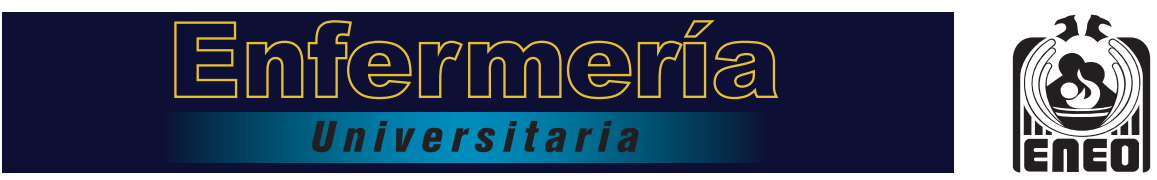

\title{
Riesgo de pie diabético y déficit de autocuidados en pacientes con Diabetes Mellitus Tipo 2
}

\section{Diabetic foot risk and self-care shortfalls among Type 2 Diabetes Mellitus patients}

\section{Risco de pé diabético e déficit de autocuidados em pacientes com Diabetes Mellitus Tipo 2}

I. Couselo-Fernández ${ }^{\mathrm{a}}$, J.M. Rumbo-Prietob*

a Gerencia de Gestión Integrada de Ferrol, Servicio Gallego de Salud, Galicia, España.
${ }^{\text {b } U n i d a d ~ d e ~ C a l i d a d . ~ G e r e n c i a ~ d e ~ G e s t i o ́ n ~ I n t e g r a d a ~ d e ~ F e r r o l, ~ S e r v i c i o ~ G a l l e g o ~ d e ~ S a l u d, ~ G a l i c i a, ~ E s p a n ̃ a . ~}$

Recibido: 10 abril 2017

Aceptado: 2 noviembre 2017

\section{Resumen}

Objetivo: Conocer los factores de riesgo de pie diabético y el nivel de conocimientos sobre autocuidados en una muestra de pacientes con diabetes mellitus tipo 2 (DM2), de un centro de salud urbano.

Método: Estudio observacional descriptivo en Atención Primaria. Intervención de enfermería relacionada con pie diabético en una muestra de usuarios diagnosticados de DM2. Recogida de datos de historia clínica, valoración clínica y aplicación de un cuestionario ad hoc sobre factores de riesgo y conocimiento de autocuidados. Análisis estadístico descriptivo e inferencial.

Resultados: 42 hombres y 31 mujeres. Media de edad de 69.1 años. El 65.8\% fueron diagnosticados de DM2 hace más de 10 años. El valor medio de la HbA1c fue de 6.9\%, el cual fue más elevado en los hombres $(\mathrm{p}=0.02)$. También, se observó mayor riesgo de complicaciones vasculares y neuropáticas en los hombres ( $\mathrm{p}=0.04)$; y una mayor presencia de deformidades (helomas y hallux valgus) en las mujeres $(\mathrm{p}<0.01)$. Déficit de conocimientos en más de la mitad de los pacientes, relacionado con la hidratación diaria de los pies $(\mathrm{p}<0.01)$ y el uso de calzado adecuado $(\mathrm{p}<0.01)$.

Conclusiones: El control de los factores de riesgo de pie diabético debe ser mejorado por parte de los pacientes de nuestro estudio. El déficit de conocimientos se relacionó con un bajo nivel informativo y con la aplicación inadecuada de las recomendaciones. Se hace necesario formar a los pacientes en estos aspectos y realizar una valoración clínica, según la práctica basada en la evidencia, lo que posiblemente influya en un mejor control del riesgo de pie diabético.

http://dx.doi.org/10.22201/eneo.23958421e.2018.1.62902

*Autor para correspondencia, correo electrónico jmrumbo@gmail.com 
Palabras clave: Pie diabético; diabetes mellitus tipo 2; pruebas diagnósticas de rutina; atención de enfermería; autocuidado; atención primaria de salud; España.

\begin{abstract}
Objective: To explore the risk factors associated with diabetic foot, and the level of knowledge on self-care, in a sample of type 2 Diabetes Mellitus patients (DM2) from an urban health center. Method: This is an observational and descriptive study in the area of nursing interventions in Primary Attention. Collected data included clinical history, clinical assessment, and the results of an ad hoc questionnaire on DM2 associated risk factors, and the self-care knowledge level. Descriptive and inferential statistics were calculated. Results: There were 42 male and 31 female patients with a mean age of 69.1 years old. 65.8\% had been diagnosed with DM2 longer than 10 years ago. The median value of HbA1c was $6.9 \%$, which was higher among males $(\mathrm{p}=0.02)$. A higher risk of suffering vascular and kidney-related complications was observed among males as well ( $p=0.04)$, whereas a higher presence of deformities such as calluses and hallux valgus was observed among females $(\mathrm{p}<0.01)$. Shortfalls in knowledge, including those on daily feet hydration $(p<0.01)$ and the use of adequate shoes $(p$ $<0.01)$ were observed in more than half of these patients. Conclusions: The control of the related risk factors must be improved by the patients in our study. Moreover, their knowledge shortfalls were related to the inadequate fulfillment of the corresponding recommendations. Thus, and considering the Evidence-Based Practice, it is necessary to better prepare these patients so that they can better control their diabetic foot risks.
\end{abstract}

Keywords: Diabetic foot; diabetes mellitus type 2; diagnostic tests, routine; nursing care; self-care: primary health care; Spain.

\title{
Resumo
}

Objetivo: Conhecer os fatores de risco de pé diabético e o nível de conhecimentos sobre autocuidados em uma amostra de diabéticos mellitus tipo 2 (DM2), de um centro de saúde urbano. Método: Estudo observacional descritivo em Atenção Primária. Intervenção de enfermagem relacionada com pé diabético em uma amostra de usuários diagnosticados de DM2. Coleta de dados de história clínica, avaliação clínica e aplicação de um questionário ad hoc sobre fatores de risco e conhecimento de autocuidados. Análise estatística descritiva e inferencial. Resultados: 42 homens e 31 mulheres. Média de idade de 69.1 anos. O 65.8\% foram diagnosticados de DM2 até mais de 10 anos. A média da HbA1c foi de 6.9\%, a qual foi mais elevada nos homens $(\mathrm{p}=0.02)$. Também, se observou maior risco de complicações vasculares e neuropáticas nos homens $(p=0.04)$; e uma maior presença de deformidades (helomas e hallux valgus) nas mulheres $(\mathrm{p}<0.01)$. Déficit de conhecimentos em mais da metade dos pacientes, relacionado com a hidratação diária dos pés $(\mathrm{p}<0.01)$ e o uso de calçado adequado $(\mathrm{p}<0.01)$. Conclusões: O controle dos fatores de risco de pé diabético deve ser melhorado por parte dos pacientes de nosso estudo. O déficit de conhecimentos relacionou-se com um baixo nível informativo e com a aplicação inadequada das recomendações. Faz-se necessário formar aos pacientes nestes aspectos e realizar una avaliação clínica, segundo a prática baseada na evidencia, o que possivelmente influencie em um melhor controle do risco de pé diabético.

Palavras chave: Pé diabético; diabetes mellitus tipo 2; testes diagnósticos de rotina; cuidados de enfermagem; autocuidado; atenção primária à saúde; Espanha. 


\section{Introducción}

La diabetes mellitus (DM) se define como un síndrome o conjunto de enfermedades caracterizadas por hiperglucemia crónica, causada por factores genéticos, epigenéticos y ambientales, que conducen a defectos en la secreción de insulina, de la acción de la insulina o a ambos ${ }^{1}$.

La clasificación actual de la DM incluye tres tipos principales de diabetes: Diabetes Mellitus tipo1 (DM1), Diabetes Mellitus tipo 2 (DM2), Diabetes gestacional; además de otros tipos específicos de diabetes ${ }^{2}$.

El diagnóstico de DM, con excepción de la presencia de síntomas clínicos, se basa en pruebas analíticas de laboratorio, consistentes en valorar las cifras plasmáticas de la glucosa anormal en ayunas (Glucemia basal en ayunas de $8 \mathrm{~h} \geq 126 \mathrm{mg} / \mathrm{dl}$; glucemia $\geq 200 \mathrm{mg} / \mathrm{dl}$ a las 2 horas de una prueba de tolerancia oral o, con síntomas de diabetes y glucemia aislada) ${ }^{1,3}$, así como, los niveles elevados de hemoglobina glicada o glicosilada o HbA1c (cifras entre 5.7 y $6.4 \%$ sirven para el diagnóstico de prediabetes y cifras $\geq 6.5 \%$ se consideran diagnósticas) ${ }^{1,4}$.

La prevalencia global en España de la DM en la población adulta ${ }^{5}$, ajustada por edad y sexo se sitúa entre un 6-13.8\%. Estas cifras representan un incremento muy importante, similar a la de todos los países del mundo. También, se ha observado un cambio significativo en el perfil o fenotipo de las personas con DM, con un significativo aumento de la DM2 (85-95\%) frente a la DM1 $(<10 \%)$, relacionado con los cambios en los estilos de vida (culturales y sociales) y en la composición corporal (obesidad, síndrome metabólico), aparecidos en las últimas décadas ${ }^{5,6}$.

Según los datos de la Federación Internacional de Diabetes ${ }^{6}$, la DM2 es el tipo de diabetes más común y está considerada como una epidemia global. Se calcula que una de cada tres personas padece DM2, cifra que varía según la edad entre un $2 \%$ a los 20 años hasta un 35\% en mayores de 65 años, ello indica la importancia de esta enfermedad conforme envejece la población. Además, el 8.3\% de la población mundial tiene DM (1 de cada 11 adultos) y dada su elevada morbilidad y mortalidad (1 de cada 10 muertes en Europa), representa en todo el mundo un grave problema de salud pública, con un importante sufrimiento humano y unos gastos sanitarios considerables (11\% del gasto) para prevenir y tratar la enfermedad y sus complicaciones ${ }^{6}$.

Otra de las consecuencias de padecer DM es que las personas con diabetes tienen un mayor riesgo potencial de desarrollar enfermedades y problemas de salud incapacitantes, invalidantes e incluso mortales, en comparación con las personas sin diabetes. Entre las complicaciones de mayor riesgo están los problemas con la mala circulación en los pies, como resultado del daño en los vasos sanguíneos. Estos problemas aumentan el riesgo de ulceración, infección y amputación ${ }^{6}$.

Bajo el concepto clínico de "pie diabético" se define una alteración clínica de base etiopatogénica neuropática, inducida por la hiperglicemia mantenida, en la que con o sin coexistencia de isquemia y previo desencadenante traumático, se produce una lesión y $/ 0$ úlcera en el pie ${ }^{7,8}$. Estas complicaciones derivadas del pie diabético suponen un sobrecoste económico al sistema sanitario, asimismo de importantes repercusiones tanto personales como sociales ${ }^{9-11}$.

La prevalencia estimada de úlceras en pie diabético (UPD) es de 1.5\%-10\%, con una incidencia de $2.2 \%-5.9 \%$. Aproximadamente, el 50\% de los pacientes mayores de 65 años diagnosticados de DM2, presentan uno o más factores de riesgo de ulceración ${ }^{7-9}$. A su vez, las complicaciones en el pie diabético suponen la primera causa de ingreso hospitalario y son el principal factor de riesgo de amputación en estos pacientes, así como la primera causa de amputación no traumática a nivel mundial ${ }^{10}$. Las personas con diabetes se enfrentan a un riesgo de amputación que puede ser 25 veces mayor que las personas sin diabetes ${ }^{6}$. En vista de estos riesgos, es importante que las personas con diabetes se examinen los pies regularmente.

La evidencia actual ${ }^{11-20}$ sugiere que se puede prevenir y/o reducir el riesgo de complicaciones por la mala circulación en los pies (ulceraciones e infecciones) con una adecuada estrategia que comprenda el cribado, la clasificación del riesgo y medidas efectivas de prevención y tratamiento. A nuestro 
entender, tales prácticas preventivas se encuadran en un Marco Estratégico para la mejora de la Atención Primaria en España ${ }^{21}$, y se justifican dentro de la atención integral que la enfermera de familia ${ }^{22}$ y comunitaria realiza al paciente crónico, desde el ámbito de la atención primaria de salud ${ }^{23}$.

Por todo ello, el objetivo de investigación fue conocer los factores de riesgo de pie diabético y el nivel de conocimientos sobre autocuidados en una muestra de pacientes con diabetes mellitus tipo 2 , adscritos a un centro de salud urbano; con la finalidad de realizar una estratificación del riesgo de pie diabético, planificar intervenciones de enfermería más eficientes de prevención, conseguir una mejor gestión de los recursos y mejorar la calidad de vida de los pacientes afectados.

\section{Material y métodos}

Se realizó un estudio observacional, descriptivo y transversal que tuvo lugar en un Centro de Salud (CS) de carácter urbano de titularidad pública. El periodo de estudio comprendió ocho meses de duración (septiembre 2013-abril de 2014). La población de referencia fueron todos los pacientes adultos con diagnóstico de DM2, adscritos al CS en el momento del estudio.

Los criterios de inclusión fueron: a) paciente adulto (mayor de edad), registrado como DM2 en la historia clínica electrónica con el código CIAP2-T90; b) participación voluntaria, previo consentimiento informado. Se excluyeron los pacientes desplazados o adscritos temporalmente al CS, así como, aquellos que no firmaron el consentimiento informado.

La captación de los pacientes se hizo por muestreo de oportunidad, entre aquellos pacientes que acudían a la cita programada de enfermería para control habitual de su diabetes (en horario de mañana y tarde), dentro del periodo del estudio.

Los pacientes que voluntariamente dieron su consentimiento para participar en el estudio, se les dio cita concertada en horario de tarde (de 15:00 a 21:00 horas), en día lectivo (de lunes a viernes), para la consulta especial de enfermería de pie diabético (creada específicamente para este estudio), para que un enfermero residente de $2^{\circ}$ año de la especialidad en enfermería familiar y comunitaria, le realizase un cribado preventivo de los factores de riesgo de pie diabético (encuesta, exploración y valoración clínica de ambos pies). En alguna ocasión se valoró la posibilidad de establecer un día para citar en horario de mañana (8:00 a 15:00 h.). El proceso de cribaje tuvo lugar en una consulta de enfermería del CS y la duración de cada sesión tuvo un promedio 20 minutos.

Por cada participante se rellenó un cuaderno de recogida de datos diseñada ad hoc, según las recomendaciones de evidencia de guías clínicas sobre pie diabético y el protocolo del centro. Las variables registradas fueron: datos sociodemográficos (sexo, edad y fecha del diagnóstico DM2), cifra de la última hemoglobina glicosilada (HbA1c), antecedentes de úlcera de pie diabético (si/no), hábito tabáquico (fumador/no fumador) y, régimen terapéutico (tratamiento farmacológico, dieta y ejercicio).

Por otro lado, existen en la actualidad 5 sistemas o escalas de estratificación de riesgo ${ }^{14}$, pero ninguna de ellas está validada en España. En nuestro estudio hemos utilizado la clasificación del International Working Group on the Diabetic Foot's (IWGDF) ${ }^{10}$, desarrollada en 2001 (modificada en 2008) a través del consenso de 45 expertos de 23 países, destaca por ser muy simple y de fácil aplicación ${ }^{10,24}$ (Tabla 1).

También, se registraron las siguientes variables relacionadas con el riesgo de neuropatía: sensibilidad protectora (Test monofilamento Semmes-Weinstein), sensibilidad profunda (Test diapasón Ryder-Seiffer), reflejo Aquileo y sensibilidad térmica, dolorosa y táctil. Para las variables de riesgo de enfermedad arterial periférica (EAP), se valoró la presencia de pulsos distales (palpación pulsos pedio y/o tibial posterior en ambos pies), Índice Tobillo-Brazo (ITB) o Índice de Yao (diferencia presión sistólica maleolar-brazo); así como, variables relacionadas con deformidades en el pie mediante la inspección, la presencia de: hiperqueratosis y/o helomas ("callos o durezas”), dedos en garra y hallux valgus (“juanete o bunio", deformidad del primer metatarso). 


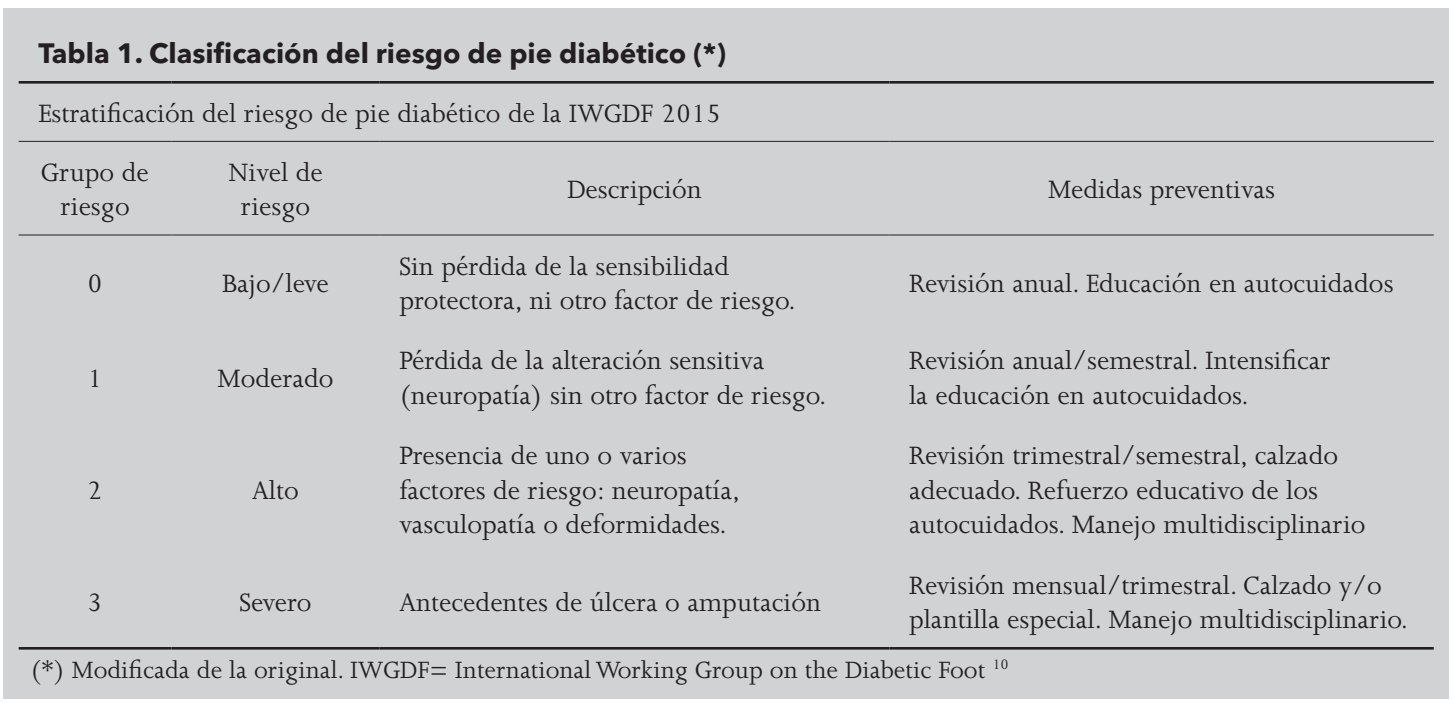

Para valorar el déficit de autocuidados se realizó un batería de preguntas ad hoc, en base a la bibliografía publicada sobre: "Conoce cuidados específicos del pie en relación a su enfermedad” (si/ no), "Ha recibido de su enfermera información relativa al cuidado de los pies" (si/no), "Higiene: Inspección diaria (si/no), lavado diario (si/no), hidratación de la piel (si/no)", "Uñas: Corte adecuado" (observación del borde de las uñas en ángulo recto), "Calzado adecuado" (calzado que lleva el paciente el día de la revisión. Se considerará calzado inadecuado: calzado en mal estado, calzado sin contrafuerte, calzado con abertura delantera o en el talón, calzado de puntera estrecha, tacón mayor de $2-3 \mathrm{~cm})$.

Con todos los datos, se realizó estadística descriptiva mediante el cálculo de frecuencias, porcentajes y medidas de dispersión (desviación estándar (DE)); y la aplicación de la estadística inferencial, para determinar el grado de asociación de las variables independientes en relación a las variables dependientes. Para el estudio analítico empleamos la comparación de medias a través de la t de Student; Ji cuadrado $\left(\mathrm{Chi}^{2}\right)$ y Test exacto de Fisher, para la comparación de proporciones. Calculamos los intervalos de confianza (IC) al 95\%. El análisis estadístico se efectuó por medio del paquete estadístico Statistical Package for the Social Sciences (SPSS) v. 19.

Este estudio de investigación contó con el correspondiente dictamen favorable del Comité Autonómico de Ética de la Investigación y las preceptivas autorizaciones de la Administración Sanitaria. Respetándose en todo momento las normas ético-legales, en particular la Declaración de Helsinki, el Convenio de Oviedo y la ley Orgánica 15/1999 de protección de datos de carácter personal.

\section{Resultados}

La muestra fue de 91 pacientes, de los que 73 participantes se consideraron válidos para el estudio. Se dio un $20 \%$ de pérdidas debido a una falta de datos en la historia clínica, abandono del propio paciente (no acude a la cita) y por cribaje incompleto (paciente no valorable). En la Tabla 2 se resumen las principales características y valores obtenidos.

En el grupo estudiado el 57.5\% fueron hombres. La media de edad se situó en 69.1 años (9.01 $\mathrm{DE})$, con un rango entre 41 y 89 años. El $11 \%$ de los participantes fueron diagnosticados de DM2 hace menos de 5 años, el 17\% entre 5-10 años y, el 65.8\% hace más de 10 años. Un 16.4\% del total de la muestra declararon ser fumadores activos. El valor medio de la HbA1c fue de 6.9\% (1.22 DE), rango entre 5.2 y $13 \%$. 
Tabla 2. Principales características de la muestra

\begin{tabular}{|c|c|c|c|}
\hline Variables & $\begin{array}{c}\text { Hombres } n=42 \\
\%\end{array}$ & $\begin{array}{c}\text { Mujeres n=31 } \\
\%\end{array}$ & $\begin{array}{c}\text { Significación } \\
\text { NS= No significativo }\end{array}$ \\
\hline Sexo & 57.5 & 42.5 & \\
\hline Edad (años) (DE) & $68.5(9.11)$ & $70.1(8.96)$ & NS \\
\hline$>65$ años & 85.7 & 90.3 & NS \\
\hline \multicolumn{4}{|l|}{ Diagnóstico DM 2: } \\
\hline$<5$ años & 11.9 & 9.7 & \multirow{3}{*}{ NS } \\
\hline 5-10 años & 21.4 & 25.8 & \\
\hline$>10$ años & 66.7 & 64.5 & \\
\hline \multicolumn{4}{|l|}{ Hábito tabáquico: } \\
\hline Fumador & 19.1 & 12.9 & \multirow[t]{2}{*}{ NS } \\
\hline No fumador & 80.9 & 87.1 & \\
\hline HbA1c (\%) (DE) & $7.09(1.46)$ & $6.68(0.70)$ & $\mathrm{P}=0.025$ \\
\hline \multicolumn{4}{|l|}{ Riesgo de Pie Diabético (IWGDF): } \\
\hline Categoría 1 & 76.2 & 93.5 & \\
\hline Categoría 2 & 2.4 & 0.0 & \\
\hline Categoría 3 & 14.3 & 0.0 & \\
\hline Categoría 4 & 7.1 & 6.5 & $\mathrm{P}=0.046$ \\
\hline Antecedentes de úlcera: & 7.1 & 0.0 & NS \\
\hline \multicolumn{4}{|l|}{ Régimen terapéutico } \\
\hline Dieta y ejercicio & 9.5 & 22.5 & \multirow{4}{*}{ NS } \\
\hline Dieta y ejercicio + ADO & 61.9 & 67.8 & \\
\hline Dieta y ejercicio + ADO + INS & 21.5 & 6.5 & \\
\hline Dieta y ejercicio + INS & 7.1 & 3.2 & \\
\hline
\end{tabular}

Según los valores obtenidos con la escala IWGDF, se observó riesgo de neuropatía en el 1.4\% de las personas; riesgo de enfermedad arterial periférica (EAP) y pérdida de sensibilidad protectora y/o deformidades en el $11 \%$. En tres casos (4\%) hubo antecedentes de úlcera de pie diabético; sin recidiva durante el periodo de estudio.

La distribución según el régimen terapéutico fue: un 15\% se controlaban por medio de dietas específicas para diabéticos y ejercicio regular, un $64.5 \%$ con dieta y ejercicio, y con antidiabéticos orales, otro 15\% con dieta y ejercicio, con antidiabéticos orales e insulina subcutánea y, finalmente, un 5.5\% con dieta y ejercicio más insulina subcutánea.

Referente a la evaluación de los factores de riesgo relacionados con la neuropatía, en la Tabla 3 se describen los valores obtenidos. Observamos que, el 90.4\% tenía la sensibilidad protectora presente y el 91.8\% la sensibilidad profunda (diapasón). El 100\% de los casos conservaba el reflejo aquíleo, la sensibilidad térmica (frío/calor plantar), la sensibilidad al dolor (presión en la uña $1^{\circ}$ dedo pie) y la sensibilidad táctil (roce con pincel en laterales del pie).

En el caso de las variables relacionadas con la EAP, en la Tabla 4 se resumen los valores resultantes. Identificamos que la palpación de pulsos distales (Pedio/Tibial posterior) estuvo presente en el 83.5\% de la muestra, cuando la palpación se hacía de forma manual. Si la toma de pulsos se realizaba con un instrumento Doppler se obtenían resultados del 100\%. Respecto al Índice Tobillo-Brazo (ITB), el 74\% de los pacientes mostraron presiones dentro de los valores considerados normales, en un $5 \%$ de los casos se detectó valores de isquemia moderada y en el $21 \%$ restante se identificó valores de isquemia leve. No hubo casos de pacientes con sospecha de calcificación arterial o de isquemia grave. En cuanto a la presencia de alguna deformidad podálica, el 56\% de los pacientes valorados presentó algún tipo de deformidad (ver Tabla 4). 
Tabla 3: Valoración de los factores de riesgo de neuropatía

\begin{tabular}{lccc}
\multicolumn{1}{c}{ Variables } & Hombres $(\mathrm{n}=42) \%$ & $\begin{array}{c}\text { Mujeres }(\mathrm{n}=31) \\
\%\end{array}$ & $\begin{array}{c}\text { Significación } \\
\text { (NS= no significativo) }\end{array}$ \\
\hline Sensibilidad protectora & 88.1 & 93.5 & NS \\
Sensibilidad profunda & 88.1 & 96.8 & NS \\
Reflejo aquíleo & 100 & 100 & NS \\
Sensibilidad térmica & 100 & 100 & NS \\
Sensibilidad dolorosa & 100 & 100 & NS \\
Sensibilidad táctil & 100 & 100 & NS \\
\hline
\end{tabular}

\begin{tabular}{lccc} 
Tabla 4. Presencia de riesgo de EAP $\mathbf{y}$ deformidades podálicas en la muestra & \\
\hline \multicolumn{1}{c}{ Variables } & $\begin{array}{c}\text { Hombres }(\mathrm{n}=42) \\
\%\end{array}$ & $\begin{array}{c}\text { Mujeres }(\mathrm{n}=31) \\
\%\end{array}$ & $\begin{array}{c}\text { Significación } \\
\text { (NS= no significativo) }\end{array}$ \\
\hline Palpación pulsos pedio-tibial posterior & 76.1 & 93.5 & $\mathrm{p}=0.04$ \\
Índice tobillo-brazo (ITB) & $0.93(0.17)$ & $0.97(0.10)$ & $\mathrm{p}<0.001$ \\
Hiperqueratosis & 40.5 & 35.5 & $\mathrm{NS}$ \\
Helomas & 14.3 & 38.7 & $\mathrm{p}=0.02$ \\
Dedos en garra & 9.5 & 9.7 & $\mathrm{NS}$ \\
Hallux valgus & 7.1 & 35.5 & $\mathrm{p}<0.001$ \\
\hline (EAP= Enfermedad Arterial Periférica; rango) & & &
\end{tabular}

Finalmente, en referencia a la evaluación de las variables relacionadas con el déficit de autocuidados, en la Tabla 5 se recogen los resultados obtenidos. El 48\% de los participantes conocen los autocuidados específicos del riesgo de pie diabético. Han recibido educación sanitaria relativa al cuidado de los pies, por parte de su enfermera (en los últimos 6 meses) el 46.5\%. Respecto a la higiene de los pies: hacen inspección diaria de los pies el 50.6\%, lavado diario el 97\% e hidratación de la piel el 45.2\%. Se cortan las uñas de forma adecuada el $65.7 \%$ de los pacientes valorados. Referente al calzado, éste fue adecuado en el $74 \%$ de los casos evaluados. Solo un 20.5\% realiza visitas frecuentes al Podólogo.

\begin{tabular}{lccc}
\multicolumn{1}{c}{ Tabla 5. Opinión de los participantes sobre los autocuidados del pie diabético } & \\
\hline \multicolumn{1}{c}{ Autocuidados } & $\begin{array}{c}\text { Hombres }(\mathrm{n}=42) \\
\%\end{array}$ & $\begin{array}{c}\text { Mujeres (n=31) } \\
\%\end{array}$ & $\begin{array}{c}\text { Significación } \\
\text { (NS= no significativo) }\end{array}$ \\
\hline Sí, conoce los autocuidados de los pies & 42.8 & 54.8 & NS \\
$\begin{array}{l}\text { Sí, recibió educación sanitaria de enfermería } \\
\text { sobre el riesgo de pie diabético }\end{array}$ & 45.2 & 48.4 & NS \\
Sí, lava a diario los pies & 97.6 & 96.7 & NS \\
Sí, inspecciona a diario los pies & 47.6 & 54.8 & NS \\
Sí, hidrata a diario los pies & 30.9 & 64.5 & p<0,001 \\
Sí, recorta adecuadamente las uñas & 59.5 & 74.2 & NS \\
Sí, presentaba calzado adecuado & 90.4 & 51.6 & NS \\
Sí, suele visitar al Podólogo & 14.2 & 19.3 & NS \\
\hline
\end{tabular}


En el análisis inferencial, el valor medio de la HbA1c fue de 6.9\%, el cual fue más elevado en los hombres $(\mathrm{p}=0.02)$; de igual forma se observó mayor riesgo de complicaciones vasculares y neuropáticas en los hombres $(\mathrm{p}=0.04)$; y una mayor presencia de deformidades (helomas y hallux valgus) en las mujeres $(\mathrm{p}<0.01)$. Déficit de conocimientos en los hombres relacionado con la hidratación diaria de los pies $(\mathrm{p}<0.01)$ y el uso de calzado inadecuado en las mujeres $(\mathrm{p}<0.01)$.

\section{Discusión}

Este estudio, sobre el cribaje de los factores de riesgo de pie diabético (o síndrome de pie diabético) en pacientes con DM2, se desarrolló como trabajo de investigación de fin de residencia dentro de la especialidad de enfermería de familia y comunitaria (EIR EFyC). A través de las intervenciones expuestas y realizadas hemos podido conocer una de las actividades y/o estrategias asistenciales, sobre una de las enfermedades crónicas más prevalentes a las que se enfrenta a diario la enfermería de familia y comunitaria (enfermera de atención primaria), para ayudar a prevenir y/o limitar los daños que produce la DM2 a nivel de los pies. El hecho de que este estudio detectase, en un 5\% de casos, valores de isquemia moderada, un $21 \%$ de isquemia leve; así como, observar que el $56 \%$ de los pacientes valorados presentó algún tipo de deformidad; indica lo importante y necesario, que la enfermería realice este tipo de cribaje del pie en personas con DM2 de forma más asidua y programada, indicador de calidad asistencial que estaría muy por debajo de lo esperado (el 100\% de los participantes no tenían registrado el cribaje realizado, a pesar de que llevaban años con la patología.

En la literatura existen diferentes métodos, técnicas y estrategias para realizar un buen cribaje de factores de riesgo de pie diabético por enfermería. En este estudio no hemos cubierto todos los recomendados (por diseño del estudio, objetivos y recursos), pero si hemos seguido la mayoría de las propuestas por las guías de práctica clínica $(\mathrm{GPC})^{7,12-19}$. La investigación actual propone, además de los recomendados en nuestro estudio, otros $\mathrm{como}^{25-38}$ : la necesidad de evaluar el hábito alcohólico y el tomar café, los niveles de colesterol HDL y LDL, la presencia de un índice de masa corporal (IMC) elevado, así como el riesgo de desarrollar retinopatía, la presencia de HTA (hipertensión arterial), la ausencia de reflejo aquíleo, rotuliano y cutáneo plantar, el aumento del umbral de la sensibilidad vibratoria, la medición de zonas de elevada presión plantar, la reducción de la movilidad (especialmente en la flexión de la columna vertebral); e incluso, realizar pruebas electrofisiológicas como la biopsia del nervio, la biopsia de la piel o la resonancia magnética de la columna espinal.

Con la batería de variables utilizadas para valorar posibles riesgos y/o complicaciones en la DM2 del síndrome del pie diabético (neuropatía periférica, enfermedad vascular periférica, úlceras, traumatismos y deformidades de los pies), metodológicamente el estudio es comparable con otros de características similares ${ }^{39-44}$, en el cual se encontraron algunos resultados relevantes (que comentamos más adelante), que hacen referencia a alteraciones de la sensibilidad protectora (Test Monofilamento), a la sensibilidad profunda (Test Diapasón Ryder-Seiffer), la disminución de pulsos pedios anteriores y tibiales posteriores, aumento del índice ITB, evolución de la diabetes, mal control de la diabetes, hábito tabáquico, presencia de deformidades podálicas y callosidades, déficit en el autocuidados de los pies y uso de calzado inapropiado.

En cuanto a las características demográficas de la muestra estudiada, la edad media (69.1 años) es bastante similar a la publicada en la literatura sobre este tema ${ }^{39,41,44}$. Sin embargo, en este estudio (la muestra se seleccionó por oportunidad, no probabilístico) existe un mayor porcentaje de varones (57.5\%), frente al predominio de mujeres que aparecen referenciados en los estudios similares ${ }^{39-44}$.

Como era de esperar, los pacientes con más años eran a su vez los más "veteranos" en el padecimiento y evolución de la enfermedad. En este estudio, a diferencia de los datos reportados por otros estudios ${ }^{41,43,44}$, los participantes tenían mayor promedio de evolución de la DM2 superior a los 10 años $(65.8 \%$ de la muestra); la explicación puede venir dada por el alto índice de envejecimiento 
poblacional (supervivencia) de nuestra zona de influencia, o por la forma de captación de los participantes (las personas mayores son las que frecuentan más veces el centro de salud).

Por otro lado, en la literatura se dan tasas del 40-70\% de pacientes DM2 catalogados como de alto riesgo para el desarrollo de úlceras ${ }^{32-35}$. En este estudio el riesgo estaría alrededor de un 16\%; así mismo, el porcentaje de individuos con antecedente de úlcera o amputación establecida fue bueno (menos del 4\%). Esto nos da una idea de que clínicamente los pacientes están bien controlados (prevención de úlceras) y que corresponde con el alto nivel de formación en el ámbito de la enfermería de la zona ${ }^{45}$.

El hábito de fumar, factor de riesgo de reconocida importancia en la aterosclerosis arterial, que en el caso de este tipo de pacientes tiende a ser una forma de presentación periférica, los valores obtenidos en este estudio son bajos (16.4\%), lo que a priori influye favorablemente en el buen estado vascular de los pacientes evaluados. La hemoglobina glicosilada (HbA1c), es un buen indicador para determinar la mala evolución de la diabetes. En este estudio el 30.9\% de los pacientes tenían cifras más altas de las recomendadas (lo normal es $<7 \%$ ), lo que nos indica un mal control de su diabetes. Las mujeres mostraron en general mejor promedio de control que los hombres, el rango entre 40 a 60 años fueron los que peores cifras de HbA1c tenían registradas. El motivo puede estar influido porque la mayoría de los pacientes eran polimedicados y la adherencia al tratamiento es un factor que no hemos tenido en cuenta.

De acuerdo al régimen terapéutico para controlar la DM2, y evaluadas las cifras de HbA1c, está justificada la variedad de tratamientos que toman los pacientes valorados en este estudio para controlar las cifras de glucemia. Los hipoglucemiantes orales (metformina y acarbosa) fueron los fármacos más utilizados como único tratamiento asociado con la dieta (71.4\% en los hombres y $89 \%$ en las mujeres), lo que coincide con las guías consultadas ${ }^{13,19}$. Sólo en algunos casos de mal control y/o evolución de la DM2, se utilizó la insulina como único tratamiento farmacológico (5\% de la muestra).

Para la valoración neurológica existen diferentes test, según la bibliografía ${ }^{7,12-19}$ se recomienda que uno sea el del monofilamento de Semmes-Weinstein y realizar, además otro de los siguientes: Diapasón de 128 Hz, Reflejo Aquíleo, Sensación dolorosa y Biotensiómetro. Para la evaluación vascular las recomendaciones indican la palpación de los pulsos pedios y tibial, así como la realización del índice tobillo-brazo. En nuestro trabajo la afectación sensitiva de la muestra evaluada obtuvo valores por debajo del $10 \%$, con base en esto se puede considerar, que el riesgo es relativamente bajo. Por el contrario, al evaluar el riesgo de la enfermedad vascular periférica (EVP), el valor del índice de Yao permitió detectar posible sospecha de isquemia leve y, en algún caso, clasificarla como isquemia moderada. Este dato aumentaría el porcentaje de riesgo de amputación, de hecho, al asociar los valores del índice tobillo-brazo (ITB) con los valores de la HbA1C, se obtuvo una $\mathrm{p}<0.01$, cuando los valores de la hemoglobina glicosilada eran mayores al $8 \%$ y la ITB presentaba algún grado de isquemia. Según la literatura publicada ${ }^{13,19}$, se cuantifica que por cada aumento del $1 \%$ de la HbA1c se produce un incremento del 25\% del riesgo de padecer EVP. En nuestro caso se dieron pacientes que estaban en ese 25\% de riesgo e incluso alguno más. Este dato valida a su vez el obtenido en la clasificación de riesgo de pie diabético, donde el 15\% de la muestra es considerada de categoría 2-3 (escala IWGDF).

Otro factor importante de riesgo de pie diabético lo constituyen la presencia de deformidades en los pies asociadas o no con lesiones cutáneas o huesos del pie. En la muestra estudiada el 56\% de los pacientes presentaban algún tipo de deformidad en algunos de sus pies. Según el tipo de deformidad se obtuvo significancia estadística de acuerdo a la variable "sexo". En general, la presencia de deformidades en ese porcentaje de muestra indica que este riesgo está presente en una alta proporción y que, de no ser corregido, a corto-mediano plazo va a suponer una complicación limitante para el paciente. Al tenor de los resultados y de la prevalencia del riesgo por este motivo, se hace necesario adecuar medidas correctivas y desarrollar estrategias, también con otros como los podólogos, profesionales infra-visitados por este tipo de pacientes, según datos del estudio solo un 20.5\% de pacientes acuden a éstos, cuando hay deformidades en el pie. 
Desde el punto de vista preventivo, el calzado desempeña un papel importante, pero puede resultar nocivo cuando no es adecuado, bien porque se encuentre en mal estado, o bien porque sus características no sean las apropiadas, que contribuyen a la deformidad del pie o a su ulceración. En este estudio se ha encontrado un calzado inadecuado en un $26 \%$ de los casos, con diversas significancias estadísticas, por un lado, al hábito de las mujeres $(\mathrm{p}<0.01)$ a usar un calzado poco adecuado, así como en los pacientes de rango 60-69 años $(\mathrm{p}<0.01)$ a usar un calzado en mal estado.

En conclusión, sobre los hábitos de autocuidado de los pies, se observó inicialmente un pobre conocimiento de los cuidados específicos de los pies para prevenir complicaciones derivadas de la DM2. Este déficit de autocuidados puede estar motivado, entre otras causas, por la poca información que reciben de los profesionales sanitarios o por su nivel cultural (factor no incluido en este estudio), el $54 \%$ de los pacientes refirieron no haber recibido educación sanitaria al respecto. Estamos de acuerdo con lo descrito en un reciente estudio de base cualitativa, donde se refleja que los profesionales sanitarios únicamente informamos de contenidos sin expresar aspectos que contribuyan a la sensibilización de los pacientes y ayudarlos a hacerse responsables de su cuidado ${ }^{46}$. Fue interesante observar variables como, la hidratación de la piel que es significativa $\mathrm{p}<0.01$, ya que sólo las mujeres lo tienen arraigado como una rutina habitual, cosa que en los hombres es un hábito poco frecuente. Igualmente, la inspección diaria de los pies (autoexploración) es realizada por un escaso 50.6\% de los pacientes. Estos datos se asocian con otras series de pacientes estudiados ${ }^{39,41,44}$.

Entre las limitaciones del estudio reseñar que la muestra se seleccionó de forma intencionada entre un grupo de pacientes que acudieron al centro de salud (sesgo de muestra). Las pérdidas de pacientes también fueron elevadas (20\%) por lo que el cálculo muestral no resulto significativo para trasladar los resultados a la población de referencia. Como sesgos de información debemos considerar la falta de medición de algunas variables por no figurar en la historia clínica del paciente, por ejemplo, la HbA1c, los antecedentes de úlcera previa, o estratificación del riesgo de pie diabético. Del mismo modo, hay que tener en cuenta que no se valoraron otras variables significativas según la bibliografía revisada, como el índice de masa corporal, la HTA, el HDL y LDL, la retinografía, hábito enólico, consumo de café, etc. El cuestionario utilizado no estaba validado y se basó en las recomendaciones de las GPC (sesgo de información).

\section{Conclusiones}

Al tenor de los resultados, así como tener en cuenta los objetivos propuestos, las limitaciones y sesgos descritos en el apartado de discusión, la conclusión fundamental de este estudio de investigación, se puede resumir en: la población estudiada está muy envejecida, y sobrelleva el diagnostico de DM tipo 2 desde un largo periodo de años. Son pacientes no fumadores en su conjunto, con una baja incidencia de úlceras o amputación de pie diabético, pero tienen potencialmente una alta prevalencia de riesgo de producir complicaciones por un mal control glucémico y unos índices de Yao (ITB), que predisponen a presentar en algún momento del periodo, episodios de isquemia leve-moderada. Otro aspecto importante a tener en cuenta, fue la falta de información sobre cuidados del pie diabético, algo esencial para su prevención.

Por tanto, la principal aplicación práctica de este estudio será poner en conocimiento del centro y sus responsables dichos datos, para luego diseñar las estrategias de salud que se estimen oportunas, así como establecer planes de actuación para la prevención del desarrollo del pie diabético desde las consultas de enfermería. Asimismo, sería necesario establecer grupos multidisciplinares, donde estén implicados otros profesionales, preferentemente podólogos, para coordinar todos estos aspectos, desde un punto de vista holístico e integral. 


\section{Responsabilidades éticas}

Protección de personas y animales. Los autores declaran que los procedimientos seguidos se conformaron a las normas éticas del comité de experimentación humana responsable y de acuerdo con la Asociación Médica Mundial y la Declaración de Helsinki.

Confidencialidad de los datos. Los autores declaran que han seguido los protocolos de su centro de trabajo sobre la publicación de datos de pacientes.

Derecho a la privacidad y consentimiento informado. Los autores han obtenido el consentimiento informado de los pacientes y/o sujetos referidos en el artículo. Este documento obra en poder del autor de correspondencia.

Financiamiento. Ninguno

Conflictos de intereses. Los autores declaran no tener conflictos de intereses

\section{Referencias}

1. American Diabetes Association (ADA). Standards of medical care in diabetes 2015: Summary of Revisions. Diabetes Care. 2015; 38 (Suppl):S4. https://dx.doi.org/10.2337/dc15-S003

2. Thomas CC, Philipson LH. Update on diabetes classification. Med Clin North Am. 2015; 99(1):116. https://dx.doi.org/10.1016/j.mcna.2014.08.015

3. Zhang X, Gregg EW, Williamson DF, et al. A1c level and future risk of diabetes: a systematic review. Diabetes Care. 2010; 33(7):1665-73. https://dx.doi.org/10.2337/dc09-1939

4. Nakagami T, Tajima N, Oizumi T, et al. Hemoglobin A1c in predicting progression to diabetes. Diabetes Res Clin Pract. 2010; 87(1):126-31. https://dx.doi.org/10.1016/j.diabres.2009.11.001

5. Soriguer F, Goday A, Bosch-Comas A, et al. Prevalence of diabetes mellitus and impaired glucose regulation in Spain: the Di@bet.es Study. Diabetología. 2012; 55(1):88-93. https://dx.doi. org/10.1007/s00125-011-2336-9

6. International Diabetes Federation (IDF). Atlas de la Diabetes. $7^{a}$ edición. Bruselas (Bélgica). IDF; 2015.

7. Asociación Española de Enfermería Vascular y Heridas. Guía de Práctica Clínica: Consenso sobre Úlceras Vasculares y Pie Diabético. Sevilla: AEEVH; 2014.

8. Verdú-Soriano J, Marinel-lo J, Armans E, et al. Conferencia Nacional de Consenso sobre Úlceras de la Extremidad Inferior. Documento de Consenso C.O.N.U.E.I. Barcelona: EdikaMed; 2009.

9. Blanes JI, Clará A, Lozano F, et al. Documento de Consenso sobre el Tratamiento de las Infecciones en el Pie del Diabético. Angiología. 2012; 64(1):31-59. https://dx.doi.org/10.1016/j. angio.2011.11.001

10. Bus SA, Van Netten JJ, Lavery LA, et al. IWGDF guidance on the prevention of foot ulcers in at-risk patients with diabetes. Diabetes Metab Res Rev. 2016; 32(1) (Suppl 1): 16-24. https://dx.doi. org/10.1002/dmrr.2696..

11. Iglesias-González R, Roura-Olmeda P. ¿Hay que realizar el cribado del pie diabético? ¿Con qué frecuencia? ¿Con qué método? (Actualización 2015). En: Ezkurra-Loiola P(Coordinador). Guía de actualización en diabetes mellitus tipo 2. Barcelona: Fundación redGDPS; 2016.

12. Salomón-Velázquez M. (Editor). Prevención, diagnóstico y tratamiento oportuno del Pie Diabético en el primer nivel de atención. $2^{a}$ Ed. México: CENETEC. Secretaría de salud; 2012.

13. Instituto Mexicano del Seguro Social (IMSS). Guía de práctica clínica GPC: diagnóstico y tratamiento de la diabetes Mellitus tipo 2. 2a ed. México: Instituto Mexicano del Seguro Social (IMSS); 2012. 
14. National Institute for Health and Clinical Excellence (NICE). Diabetic foot problems. Inpatient management of diabetic foot problems. London: NICE; 2011.

15. Australian Wound Management Association (AWMA). Prevention, Identification and Management of Foot Complications in Diabetes. National Evidence-Based Guideline. Melbourne, Australia: Commonwealth of Australia; 2011.

16. Registered Nurses' Association of Ontario (RNAO). Assessment and Management of Foot Ulcers for People with Diabetes. Clinical Best Practice Guidelines. Ontario (Canada): RNAO; 2013.

17. Scottish Intercollegiate Guidelines Network (SIGN). Management of diabetes. A national clinical guideline. Edinburgh: SIGN; 2010.

18. Hingorani A, LaMuraglia GM, Henke P, et al. The management of diabetic foot: A clinical practice guideline by the Society for Vascular Surgery in collaboration with the American Podiatric Medical Association and the Society for Vascular Medicine. JVasc Surg. 2016; 63 (2 Suppl):3S-21S. https:// dx.doi.org/10.1016/j.jvs.2015.10.003

19. Grupo de Trabajo de la Guía de Práctica Clínica sobre Diabetes Tipo 2. Guía de Práctica Clínica sobre Diabetes Tipo 2. Madrid: Plan Nacional para el SNS del MSC. Agencia de Evaluación de Tecnologías Sanitarias del País Vasco; 2008.

20. International Diabetes Federation (IDF) Working Group. International Diabetes Federation managing older people with type 2 diabetes. Global guideline. Brussel (Belgium): IDF; 2013.

21. Melguizo Jiménez M, Cámara González C, Martínez Pérez JA, et al. Marco Estratégico para la mejora de la Atención Primaria en España: 2007-2012. Proyecto AP-21. Madrid: Ministerio de Sanidad y Consumo, 2007.

22. Asociación Andaluza de Enfermería Comunitaria (ASANEC). Guía de atención enfermera a personas con diabetes. Granada: ASANEC; 2004.

23. Ministerio de Sanidad y Política Social. Orden SAS/1729/2010, de 17 de junio, por la que se aprueba y publica el programa formativo de la especialidad de Enfermería Familiar y Comunitaria. Madrid: Boletín Oficial del Estado (BOE), nº 157. (29 de junio de 2010).

24. Monteiro-Soares M, Boyko EJ, Ribeiro J, et al. Risk stratification systems for diabetic foot ulcers: a systematic review. Diabetologia. 2011; 54(5):1190-9. https://dx.doi.org/10.1007/ s00125-010-2030-3.

25. Vázquez San Miguel F, Mauricio Puente D, Viadé Julià J. Neuropatía diabética y pie diabético. Medicine. 2016; 12(17):971-81

26. Botas M, Cervell D, Rodríguez Montalbán AI, et al. Actualización en el diagnóstico, tratamiento y prevención de la neuropatía diabética periférica. Angiología. 2016. https://dx.doi.org/10.1016/j.angio.2016.06.005

27. Tardáliga García A. Influencia del grado de neuropatía diabética en el grosor de la fascia plantar, las deformidades digitales y la limitación de la movilidad articular en el Pie Diabético no ulcerado. REDUCA. 2015; 7 (3):1-57.

28. Sacco IC, Hamamoto AN, Tonicelli LM, et al. Abnormalities of plantar pressure distribution in early, intermediate, and late stages of diabetic neuropathy. Gait Posture. 2014; 40 (4):570-4. https:// dx.doi.org/10.1016/j.gaitpost.2014.06.018.

29. García Álvarez Y. Identificación de las características del calzado, que desarrollan un factor de riesgo de ulceración en pacientes diabéticos con neuropatía. REDUCA. 2010; 2(1): 391-427.

30. López Fernández R, Yanes Seijo R, Suárez Surí P, et al. Modelo estadístico predictivo para el padecimiento de pie diabético en pacientes con diabetes mellitus tipo II. Medisur. 2016. 14(1): 42-52.

31. Boulton AJ. The pathway to foot ulceration in diabetes. Med Clin North Am. 2013; 97 (5):775-90. https://dx.doi.org/10.1016/j.mcna.2013.03.007 
32. Martínez-Alberto CR. Diseño y validación de un procedimiento enfermero de cribado de neuropatía diabética en atención primaria. [Tesis Doctoral]. Santa Cruz de Tenerife: Universidad de La Laguna; 2016.

33. Reverter Calatayud JL. Pie diabético. Abordaje multidisciplinar. FMC. 2015; 22(5):242-9. https:// dx.doi.org/10.1016/j.fmc.2015.02.021

34. Alfayate M, Sánchez Ríos JP, de Benito L, et al. Revisión de las recomendaciones actuales y protocolos de actuación en prevención del pie diabético. Angiología. 2012; 64:76-81.

35. Pavia O, Rojas N. Pie diabético: ¿Podemos prevenirlo?. Rev Med Clin Condes. 2016; 27 (2): 227-34.

36. Gómez-Calvache YE. Factores de riesgo asociados a la diabetes mellitus tipo 2 en el municipio de Santa Rosa, departamento de Cauca. [Tesis Máster]. Argentina: Universidad Nacional de la Plata; 2014.

37. Bermúdez V, Salazar J, Rojas J, et al. Prevalencia de diabetes mellitus tipo 2 y factores de riesgo asociados en la ciudad de Maracaibo, Venezuela. Rev Latinoam Hipertensión. 2014; 9(2): 14-25.

38. Pérez-Pevida B, Llavero M, Gargallo J, et al. Complicaciones microvasculares de la diabetes. Medicine (Barc). 2016; 12(17):958-70. https://doi.org/10.1016/j.med.2016.09.003

39. Lázaro A, Febles Sanabria R, Moliner Cartaya M. Identificación de los factores de riesgo que conducen al desarrollo de las lesiones neuropáticas ulceradas. Rev Cubana Angiol Cir Vasc. 2016; 17(1): 13-24.

40. Pinilla AE, Sánchez AL, Mejía A, et al. Actividades de prevención del pie diabético en pacientes de consulta externa de primer nivel. Rev Salud Pública. 2011; 13 (2): 262-73.

41. Alonso-Fernández M, Mediavilla-Bravo JJ, López-Simarro F, et al. Evaluación de la realización del cribado del pie diabético en Atención Primaria. Endocrinol Nutr. 2014; 61(6):311-7. http:// dx.doi.org/10.1016/j.endonu.2014.01.007

42. Márquez-Godíneza SA, Zonana-Nacachb A, Anzaldo-Camposay MC, et al. Riesgo de pie diabético en pacientes con diabetes mellitus tipo 2 en una unidad de medicina de familia. Semergen. 2014; 40(4):183-8.

43. Silva PL, Rezence M, Ferreira L, et al. Cuidado de los pies: el conocimiento de las personas con diabetes mellitus inscritos en el programa de salud familiar. Enf Global. 2015; 1(37): 38- 51. https://dx.doi.org/10.6018/eglobal.14.1.170401

44. Rossaneis MA, Fernández Lourenço Haddad MC, de Freitas Mathias TA, et al. Diferencias entre mujeres y hombres diabéticos en el autocuidado de los pies y estilo de vida. Rev. Latino-Am Enfermagem. 2016; 24: e2761. https://dx.doi.org/10.1590/1518-8345.1203.2761

45. Castro-Meza AN, Pérez-Zumano SE, Salcedo-Álvarez RA. La enseñanza a pacientes con diabetes: significado para profesionales de enfermería. Enferm Univ. 2017; 14(1):39-46. http://dx.doi. org/10.1016/j.reu.2016.12.003 